\title{
Rechazar una invitación y negarse a una petición: procedimientos de atenuación discursiva empleados en las respuestas escritas de alumnas de español como lengua extranjera en Estados Unidos
}

Declining an invitation and refusing a request: discursive attenuation procedures used in the written answers of students of Spanish as a foreign language in the United States

\section{Francisco J. Rodríguez Muñoz \\ Universidad de Almería \\ España}

Dimitrinka G. Níkleva

Universidad de Granada

España

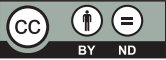

Francisco J. Rodríguez Muñoz: Departamento de Educación, Área de Didáctica de la Lengua y la Literatura, Universidad de Almería, España. | Correo electrónico:frodriguez@ual.es 


\section{Resumen}

Este artículo se propone analizar los procedimientos de atenuación discursiva que utilizan 52 alumnas de español como lengua extranjera de Estados Unidos. A partir de un cuestionario, se recogieron las respuestas en relación con dos funciones comunicativas presentes en el Plan curricular del Instituto Cervantes. En la primera, las participantes tenían que declinar la invitación de un amigo, en tanto que en la segunda, debían responder a la petición de un desconocido negándose a su cumplimiento. Aunque, inicialmente, se suponía que una mayor distancia social implicaría un mayor número de recursos de atenuación en el plano enunciativo, los resultados sugieren que existen otros factores que impiden la confirmación de dicha hipótesis. Con todo, se presentan diferencias en la cantidad y el tipo de mecanismos atenuadores vinculados a ambas funciones en dos niveles intermedios de dominio de español (uno entre $A 2$ y $B 1$, y otro entre $B 1$ y $B 2$ ).

Palabras clave: atenuación discursiva; español como lengua extranjera; negarse a una petición; rechazar una invitación.

\section{Abstract}

This paper aims to analyse discursive attenuation procedures that 52 students of Spanish as a foreign language of United States use. From a questionnaire, the answers linked to two communicative functions present in the Plan curricular del Instituto Cervantes were collected. In the first, participants had to decline an invitation from a friend, while, in the second, they had to refuse a request from a stranger. Although, initially, it was supposed to find a greater number of attenuation resources associated to participants' responses when these had relation to a larger social distance degree between interlocutors, results suggest that there are other factors that prevent the validation of this hypothesis. However, differences in the amount and type of attenuation mechanisms are found regarding both functions in two groups at an intermediate level of Spanish (the first one, between $A 2$ and $B 1$, and the second one, between $\mathrm{B} 1$ and $\mathrm{B} 2$ ).

Keywords: discursive attenuation; Spanish as foreign language; refusing a request; declining an invitation. 


\section{Introducción}

El presente trabajo se ciñe al análisis de los procedimientos de atenuación en los textos escritos de estudiantes de español como lengua extranjera (en adelante, ELE). Tanto la atenuación como, en el polo opuesto, la intensificación discursiva son conceptos indefectiblemente ligados a la investigación sobre (des)cortesía. En tales estudios se ha destacado con mayor intensidad: (1) el plano lingüístico o enunciativo (prisma pragmalingüístico); (2) el sociocultural o contextual (prisma sociopragmático); o, incluso, (3) el individual o cognitivo (prisma psicopragmático). A pesar de que debemos reconocer que estos tres ejes aparecen completamente engranados en la práctica discursiva, este artículo se centra en los recursos atenuantes que intervienen en el plano lingüístico y del enunciado. Más específicamente, nos planteamos las siguientes cuestiones:

1. ¿La mayor actividad atenuadora en el plano discursivo se corresponde con una también mayor distancia social entre el enunciador y su destinatario?

2. ¿Existen diferencias cuantitativas y cualitativas entre los niveles A2-B1 y B1-B2 de ELE al emplear procedimientos de atenuación discursiva vinculados a las funciones rechazar una invitación y responder a una petición negativamente?

A partir de estas preguntas de investigación, se formulan las siguientes hipótesis:

1. Teniendo en cuenta la mayor proximidad social que se presupone en la situación comunicativa planteada en "(1) Un amigo tuyo te pregunta si quieres ir al cine con él. ¿Cómo le dices que no?", se espera que, cuantitativamente, los procedimientos de atenuación discursiva empleados en los enunciados de nuestras participantes sean inferiores en relación con el supuesto "(2) Un desconocido te pide dinero por la calle. ¿Cómo le dices que no puedes darle nada?”.

2. Es previsible que sean más numerosos y más elaborados los recursos de atenuación discursiva utilizados conforme se vayan alcanzando niveles superiores de dominio lingüístico de ELE.

\section{Marco teórico}

\subsection{La atenuación discursiva en el contexto de la modalidad}

Tradicionalmente, se han distinguido dos componentes del enunciado: el dictum (o el contenido) y el modus (o la actitud del hablante). Este segundo concepto nos conduce a otro de gran calado en el ámbito de los estudios pragmáticos: la modalidad. Una distinción básica es la que establece Traugott (1989), quien considera la modalidad deóntica, que se relaciona con 
los deberes y las obligaciones, y la epistémica, asociada al grado de compromiso con respecto a la verdad de la proposición enunciada, o al grado de certidumbre o incertidumbre que se tiene sobre el conocimiento enunciado; esto es, los hechos son seguros o dudosos, o bien reales, probables, posibles o irreales.

Lyons (1977) añade una tercera modalidad, la alética, que se refiere a la contingencia o necesidad de tales hechos. Más aún, hay quienes incluyen una cuarta modalidad, la denominada axiológica (o apreciativa), que trasluce la actitud del enunciador con respecto a lo enunciado de acuerdo con su propia escala de valores, es decir, le permite realizar una valoración de carácter moral o estético (véase Martín Peris y otros (2008)).

Centrándonos en la atenuación discursiva, Mendiluce (2005) señala diversos valores pragmáticos asociados a determinadas palabras o expresiones que podemos adscribir aquí a un tipo u otro de modalidad, teniendo en cuenta que las fronteras entre las distintas modalidades son a veces difusas o imprecisas:

- Aproximación: se trata de atenuadores epistémicos, por cuanto disminuyen el grado de certeza o seguridad a propósito del conocimiento enunciado: tendrá casi / alrededor de / más o menos / aproximadamente sesenta años.

- Posibilidad, probabilidad o duda: estos atenuadores mitigan, asimismo, el grado de certidumbre, por lo que serían de tipo epistémico: puede ser que / probablemente / posiblemente / quizá(s) Ilueva; a lo mejor llueve; parece que va a llover. Nótese que el grado de epistemicidad del atenuador probablemente difiere del que le corresponde a posiblemente, esto es, el grado de seguridad es mayor en el primer caso que en el segundo. El uso del subjuntivo, el modo verbal vinculado a la expresión de la subjetividad por antonomasia (junto con los tiempos futuro y condicional de indicativo), ya supone un mecanismo de atenuación en sí mismo: el enunciado declarativo pudiera llover entraña una mayor inseguridad con respecto al hecho enunciado en puede llover. De igual modo, el par _ ¿Podría decirme cuánto es? - Serían cinco euros presenta un grado de atenuación mayor que el del par — ¿Me dices cuánto es? —Son cinco euros. En cuanto al verbo parecer, no solo está ligado a la modalidad epistémica, sino que podría extenderse a la axiológica cuando se trata de opinar: parece alemán.

- Relatividad o limitación en la validez de los datos: nuevamente, los relativizadores (verbos intelectivos como creer, pensar, suponer, deducir o interpretar, o determinados marcadores del discurso) sirven para establecer el grado de certeza que se tiene sobre algo, pero también son modalizadores axiológicos por medio de los cuales el enunciador da a conocer su opinión: pienso / creo que / en mi opinión, / según sé, / hasta donde sé, no vendrá.

Otros mecanismos de atenuación pragmática que indica Mendiluce (2005) son los siguientes: 
- Indeterminación: algunos cuantificadores indefinidos como algo, cierto o un poco.

- Despersonalización a través de construcciones impersonales y en voz pasiva: podemos establecer distintos grados de intensificación/atenuación, desde los dativos éticos con función intensificadora (Mi hijo me come muy bien), pasando por el uso de la primera persona del singular (yo) o por el plural de modestia (nosotros), perfectamente posible, por ejemplo, en la enunciación científica en el ámbito hispano (según nuestros datos), hasta las construcciones impersonales y en voz pasiva, más recomendables aún en el estilo académico (Regueiro y Sáez, 2015).

- Negación de intensificadores (esta estrategia atenuadora también se denomina lítote): no es seguro, no es evidente, no está claro.

La sufijación apreciativa (aumentativos, diminutivos y despectivos) es otro procedimiento lingüístico - concretamente morfopragmático (proveniente de la morfología léxica o derivativa) - que debemos incluir en el ámbito de la modalidad, pues sirve para expresar subjetivamente el afecto o el desagrado (Rodríguez Muñoz, 2012).

Debemos destacar la existencia de determinadas clases de palabras que comparten su carácter valorativo y, en ese sentido, han de relacionarse con la modalidad axiológica. Nos referimos, por ejemplo, a adverbios y locuciones adverbiales como lamentablemente, afortunadamente, por suerte o por desgracia. Sin cambiar de categorías léxicas, pero asociadas a la modalidad alética, encontramos necesaria o innecesariamente o, a la deóntica, obligatoria u opcionalmente. El concepto de modalidad también se ha aplicado, en la gramática tradicional, a los tipos de perífrasis que sirven para expresar valores subjetivos:

- Probabilísticas (modalidad epistémica): poder + infinitivo, deber de + infinitivo.

- Obligativas (modalidad deóntica): tener que + infinitivo, haber que + infinitivo, haber de + infinitivo, deber + infinitivo.

Por último, podríamos seguir añadiendo un sinfín de categorías a la lista de procedimientos lingüísticos que sirven para expresar modalidad: las figuras retóricas, la tematización, la entonación en la modalidad oral o el uso pragmático de los signos de puntuación y de las marcas tipográficas en la escrita.

\subsection{La distancia social y la variación pragmática}

Basándonos en Félix-Brasdefer (2004) y en Nejström (2012), el instrumento que empleamos en este trabajo para la recogida de datos considera dos tipos de interlocutores: conocidos -es decir, existe poca distancia social en la enunciación-, y desconocidos - existe una mayor 
distancia social en la enunciación-. En consecuencia, se distinguen dos niveles de distancia social que suponemos que condicionarán la presencia de un mayor o menor número de atenuantes en las respuestas escritas de los estudiantes de ELE en el plano enunciativo. Más específicamente, prevemos que a una mayor relajación social en la enunciación le corresponde, cuantitativamente, una menor presencia de procedimientos mitigadores en los enunciados que a una menor relajación social, que se traduciría en una relajación lingüística también inferior y, en tal caso, esperamos que los mecanismos de atenuación sean superiores. Dicho de otro modo, la relajación social, que viene dada por la situación comunicativa y por el grado de coloquialidad o formalidad que esta exige, sería inversamente proporcional a la cantidad de atenuantes presente en la actividad discursiva. En esta línea, Briz y Albelda (2013: 294) reconocen que "a mayor coloquialidad, en principio, menor actividad atenuadora".

Precisamente, Briz y Albelda (2013: 293) entienden por variación lingüística los cambios que pueden experimentar "Ios registros o estilos de comunicación” en virtud de la situación. Así, entre los aspectos que condicionan el nivel de coloquialidad o formalidad ${ }^{1}$ destacan los asociados:

a) a la mayor o menor relación de igualdad social o funcional entre los interlocutores que participan en la interacción (estratos sociales y roles), b) a la mayor o menor relación vivencial de proximidad (saberes compartidos, acercamiento interpersonal) entre los interlocutores, c) al marco o espacio interaccional más o menos cotidiano, d) a la mayor o menor cotidianidad temática del evento comunicativo, y e) al fin más o menos interpersonal. A su vez, a mayor o menor presencia de estos rasgos se asocian, respectivamente, f) un grado mayor o menor de planificación sobre la marcha, y g) un tono más o menos informal.

\subsection{Procedimientos de atenuación discursiva}

Briz (2012), remitiéndose principalmente a la modalidad oral, identifica un número elevado de procedimientos de atenuación que pueden utilizar los usuarios en su discurso²:

1. Modificadores morfológicos internos: sufijos (diminutivos).

2. Modificadores externos: cuantificadores, aproximativos, difusores de significado (un poco, algo así, más o menos, y eso, en principio, de alguna manera).

1 En la terminología de Oesterreicher (1996), de mayor o menor inmediatez comunicativa.

2 Véase también Cestero (2012), que separa los mecanismos de atenuación del dictum de los que afectan al modus, y Níkleva (2015), que sintetiza distintos recursos para expresar cortesía verbal. Asimismo, destacan los trabajos de Albelda y Cestero (2011), Albelda (2013) y Albelda y otros (2014) sobre los recursos de atenuación en español y su categorización funcional. 
3. Términos léxicos o expresiones más suaves en el contenido significativo: lítotes, eufemismos.

4. Empleo de palabras extranjeras (por ejemplo, la expresión ser de la "jet set" puede usarse para llamar rico a alguien en sentido peyorativo) u onomatopeyas (puaj, para expresar asco; puf, para denotar molestia o repugnancia; o uf, para manifestar cansancio).

5. Usos dislocados de los tiempos y modos verbales (imperfecto o condicional de cortesía).

6. Empleo de verbos y partículas discursivas con valor modal que expresan opinión en forma de duda o posibilidad (creer, parecer, ser posible, ser conveniente, quizás, posiblemente).

7. Empleo de verbos, adverbios y construcciones verbales que expresan fingimiento de incertidumbre, de incompetencia o de ignorancia (no saber, no estar seguro, no soy la persona más indicada).

8. Construcciones parentéticas que acotan la opinión a la propia persona o al espacio personal (para mí, en mi opinión, por lo menos en mi pueblo, creo yo, digo yo).

9. Peticiones, preguntas, mandatos u órdenes expresados de forma indirecta.

10. Expresiones de disculpa ante una interrupción, una pregunta, un favor o ante la expresión de una opinión (perdona que te moleste; si no te molesta, ponme un poco de café; siento interrumpir).

11. Modificaciones del acto de habla que restringen el alcance de lo dicho por el propio hablante: estructuras concesivas, temporales y condicionales (cuando quieras, me lo puedes traer; si no me equivoco, esto fue así; aunque sea tarde, puedes venir a casa).

12. Justificaciones, excusas: es que, lo que pasa es que...

13. Elipsis de la conclusión (estructuras suspendidas y truncadas).

14. Partículas discursivas y otras expresiones de control de contacto.

15. Impersonalizaciones.

16. Movimientos de reformulación (estás un poquito gorda; o sea, te sobra algún kilito).

17. Movimientos concesivo-opositivos (sí/vale/bueno, pero...).

18. Concesividad sin oposición (bueno, nos vemos mañana).

19. Empleo de elementos paralingüísticos y kinésicos: prosodia33, risas, movimientos y gestos.

20. Uso de formas de tratamiento y formas apelativas convencionalizadas (usted, oye, mira, nombre, mujer).

Briz y Albelda (2013) agrupan las tácticas o recursos lingüísticos de atenuación en dos grandes bloques que aquí sintetizamos y adaptamos:

3 Para el análisis de la atenuación prosódica en español, véase Hidalgo Navarro (2006). 
1. La ocultación del yo/tú o de terceros (indeterminación, impersonalización, despersonalización o desagentivación) a través de los siguientes recursos:

a. Formas gramaticales impersonales (se, uno, nosotros como plural de modestia, el tú impersonal).

b. Expresiones generalizadoras (todo el mundo, según dicen...).

c. Construcciones que esconden el agente de la acción (nominalizaciones como el análisis de los datos en vez de analicé los datos; pasivas sin agente explícito; y pasivas reflejas).

d. Construcciones nominales con adjetivo relacional que se alejan eufemísticamente de un término indirecto (inmigrantes irregulares por inmigrantes ilegales o asistente sexual por prostituta).

2. La relativización o indeterminación de lo expresado (recursos relativizadores) por medio de los recursos que se indican a continuación:

a. Verbos que expresan valores modales epistémicos (no saber, parecer, pensar o creer).

b. Marcadores del discurso (modalizadores como en mi opinión, quizá o a lo mejor; controladores del contacto como oye, mira, hombre o mujer; comprobativos como ¿no? y ¿eh?4).

c. Uso de tiempos verbales y construcciones hipotéticas (sea el condicional, sea el imperfecto de indicativo o subjuntivo - querría, quería, quisiera-).

d. Estructuras causales explicativas o justificativas, temporales, condicionales, concesivas (movimientos concesivo-opositivos del tipo bueno, pero...).

e. Construcciones indirectas, incluidas las construcciones suspendidas o truncadas que eluden o eliden la conclusión.

f. Movimientos de reformulación: Te equivocas; bueno, no tienes del todo razón.

g. Uso del diminutivo (estáis distraidillos), cuantificadores y partículas (más o menos, aproximadamente, como, en algunos casos, poco) que se incluyen entre los aproximadores o difusores del significado: las piedras me parecen como cosas de algodón ${ }^{5}$.

h. Proformas deícticas con significado vago o impreciso: ahí (la calle esa está por ahi), allí, así, algo así, y eso.

i. Eufemismos, fenómenos de lítote: No está bien en lugar de Está mal.

4 Sobre las funciones pragmático-discursivas de ¿no? y ¿eh?, véase Rodríguez Muñoz (2009).

5 Sobre el valor pragmático del aproximativo como, véase Gómez Mesas y Rodríguez Muñoz (2014). 
En este afán por categorizar los tipos de atenuantes y sus funciones corteses, Álvarez Muro y Joven Best (2005) (citados en Ramos González, 2015) sugieren las siguientes clases, que aquí sintetizamos:

1. Atenuación de la locución: mediante el uso de perífrasis, cuantificadores como muy, más o poco, diminutivos, negaciones de adjetivos en grado positivo en lugar de sus antónimos o eufemismos.

2. Atenuación de la ilocución: a través de marcadores como bueno, expresiones matizadoras como se puede decir, marcas de interacción como ¿verdad?, recurriendo al tiempo condicional y al modo subjuntivo, a la evasión, a las explicaciones, al no saber o al no haber estado.

3. Atenuación del origen del enunciado: se desfocaliza la fuente del enunciado, por ejemplo, usando los indefinidos (uno) o mitigando la responsabilidad del hablante por medio del se impersonal o de otras expresiones impersonales (la gente, las personas, alguien).

Por último, Kebrat-Orecchioni (2004: 49) distingue cuatro categorías en función de las marcas de cortesía presentes o ausentes en los enunciados lingüísticos:

1. Descortesía: ausencia "anormal” de un marcador de cortesía (o presencia de un marcador demasiado débil).

2. Acortesía: ausencia "normal” de un marcador de cortesía.

3. Cortesía: utilización de un marcador de cortesía más o menos esperado en el contexto.

4. Supercortesía: presencia de un marcador excesivo en relación a las expectativas normativas vigentes.

\section{Las funciones rechazar una invitación y responder negativamente a una petición en el Plan curricular del Instituto Cervantes}

Dado que las participantes de esta investigación son estudiantes de ELE que se encuentran, por un lado, entre los niveles A2 y B1, y, por otro, entre el B1 y el B2, seguidamente se reproducen los exponentes (o microfunciones) que recoge el Plan curricular del Instituto Cervantes (en adelante, PCIC) (Instituto Cervantes, 2006), a propósito de las funciones comunicativas que analizamos: rechazar una invitación —en la terminología del PCIC, Rechazar una propuesta, ofrecimiento o invitación - (tabla 1) y responder negativamente a una petición - en el mismo documento, Responder a una orden, petición o ruego > Negándose a su cumplimiento- (tabla 2). En concreto, nos interesan los exponentes asociados a los dominios A2, B1 y B2 de ELE. 


\section{TABLA 1}

Exponentes funcionales en el PCIC asociados a la función rechazar una invitación

\section{A2}

\section{B1}

B2

$\left(N_{0},\right)(n o),\left(\right.$ muchísimas) gracias $\left(N_{0},\right)(n o$,$) (muchísimas) gracias$

+ valoración + pero...

+ excusa o explicación

(No,) lo siento

+ valoración + pero...

+ excusa o explicación
+ es que...

(No,) lo siento + es que...

Pues/Bueno, es que...

Pues no.

No puedo y lo siento (de verdad).

Prefiero... + excusa o explicación.
Eres muy amable, pero (desgraciadamente) no puedo.

Lo lamento, pero...

(Lo lamento, pero / Me temo que) es / va a ser imposible + excusa o explicación (Lo lamento, pero / Me temo que) no es / va a ser posible + excusa o explicación (Lo lamento, pero / Me temo que) no voy a poder. Tengo que decirte que no. ¿Qué rabia! Te tengo que decir que no porque ya tengo otros planes.

Preferiría + contrapropuesta.

\section{TABLA 2}

Exponentes funcionales en el $P C I C$ asociados a la función responder negativamente a una petición

\begin{tabular}{|c|c|c|}
\hline $\mathrm{A} 2$ & B1 & B2 \\
\hline $\begin{array}{l}\text { De forma cortés } \\
\text { Lo siento, pero... } \\
\text { No puedo + justificación. } \\
\text { De forma tajante } \\
\text { No quiero/puedo + inf. }\end{array}$ & $\begin{array}{l}\text { De forma cortés } \\
\text { Me encantaría, pero... } \\
\text { No puedo, es que... } \\
\text { Lo siento, pero es que... } \\
\text { Lo siento, pero va a ser } \\
\text { imposible. } \\
\text { De forma tajante } \\
\text { No pienso + inf. }\end{array}$ & $\begin{array}{l}\text { De forma cortés } \\
\text { Lo lamento, pero (es que)... } \\
\text { Desgraciadamente/ } \\
\text { Lamentablemente..., (es que)... } \\
\text { De forma tajante } \\
\text { Me niego (rotundamente) a + inf. } \\
\text { No tengo por qué hacerlo. } \\
\text { No tengo ninguna obligación de } \\
\text { hacerlo. } \\
\text { No me da la gana (+ de + inf.) } \\
\text { De ninguna manera / De ningún modo. }\end{array}$ \\
\hline
\end{tabular}

Otro capítulo al que el PCIC nos remite al tratar sobre las funciones que se analizan en este trabajo es el que lleva por título "Tácticas y estrategias pragmáticas". Como subraya Llamas Saíz (2010), este apartado redunda en la necesidad de la enseñanza-aprendizaje de los recursos lingüísticos que permiten transmitir e interpretar eficazmente una intención determinada y 
alcanzar, de esa manera, el éxito en la comunicación. Precisamente, uno de los tres epígrafes de los que se compone este apartado se refiere a la "Modalización”, que, entre otros aspectos, recoge los concernientes a los procedimientos de atenuación e intensificación discursivas.

\section{Metodología 4.1. Participantes}

En la investigación participaron 52 alumnas (todas ellas, mujeres) de ELE que, durante el año académico 2015-2016, estaban matriculadas en The Spence School (Nueva York, Estados Unidos) en la etapa correspondiente al Upper School (grados 9 a 12). Las edades de estas alumnas estuvieron comprendidas entre los 14 y los 19 años - la edad modal se situó en los 14 años-. 48 señalaron que tenían nacionalidad estadounidense, mientras que 4 de ellas manifestaron poseer, además de esta, las nacionalidades china (2), coreana (1) y japonesa (1). En este último caso, se trataba de alumnas bilingües que, salvo en el contexto familiar, utilizaban el inglés para comunicarse en su vida cotidiana. Todas las estudiantes residían en el estado norteamericano de Nueva Jersey en el momento de la recogida de datos.

En cuanto al nivel de español, es necesario aclarar que el profesor de español que asistió en la recogida de datos distribuyó a las 52 participantes seleccionadas en dos grupos, que se ajustaron, por una parte, a los niveles A2-B1 —alumnas que poseen el nivel A2 y que están finalizando su preparación para el B1 - (grupo 1) y, por otra, a los niveles B1-B2 — alumnas que disponen del B1 y que están a punto de optaral B2- (grupo 2) establecidos en el Marco común europeo de referencia (Consejo de Europa, 2002 [2001]). La cantidad de alumnas por grupo se repartió del siguiente modo, en función de la disponibilidad: 29 alumnas del primer grupo y 23 alumnas pertenecientes al segundo.

\subsection{Procedimiento}

Los datos fueron recogidos presencialmente por el profesor de español de las participantes a través de un cuestionario. Se les avisó que se trataba de una investigación a cargo de dos universidades españolas, garantizando en todo momento el anonimato de las alumnas, a las que se les pidió que contestaran a las preguntas en clase sin diccionario ni ningún otro tipo de apoyo. No se les impusieron limitaciones temporales para completar la actividad.

\subsection{Instrumento}

El instrumento empleado para la recogida de datos fue un cuestionario. Para diseñarlo, se tuvieron en cuenta seis funciones comunicativas incluidas en el PCIC: (1) rechazar una invita- 
ción; (2) responder a una petición negativamente; (3) dar una opinión; (4) valorar; (5) pedir un objeto; y (6) pedir un favor.

De acuerdo con los objetivos de este trabajo, en esta ocasión, exclusivamente se prestará atención a los supuestos (1) y (2) que plantea el cuestionario. En concreto, la cuestión (1) se vincula a la función (1) rechazar una invitación, y entre los interlocutores existe poca distancia social (son amigos); la cuestión (2) se vincula a la función (2) responder a una petición negativamente, y la distancia social entre los interlocutores es alta (el usuario se dirige a un desconocido):

Supuesto 1. Un amigo tuyo te pregunta si quieres ir al cine con él. ¿Cómo le dices que no? Supuesto 2. Un desconocido te pide dinero por la calle. ¿Cómo le dices que no puedes darle nada?

Las preguntas de la encuesta fueron planteadas en dos idiomas, tanto en español como en inglés, para facilitar su comprensión. Aun así, se resaltó tipográficamente en el documento que las cuestiones se tenían que responder solamente en español.

\section{Resultados}

\subsection{Recursos de atenuación discursiva en el supuesto 1: rechazar la invita- ción de un amigo}

En este apartado se atiende a la presencia de procedimientos de atenuación discursiva en torno a la función comunicativa rechazar una invitación, que, en este caso, procede de un amigo: “(1) Un amigo tuyo te pregunta si quieres ir al cine con él. ¿Cómo le dices que no?”.

\subsubsection{Grupo A2-B1}

En el 34,5\% de las respuestas al supuesto 1 , se responde negativamente y sin ninguna marca de atenuación a la invitación de un hipotético amigo; en tales casos, podríamos interpretar que nos encontramos ante actos acorteses o descorteses - mientras que, en la segunda opción, se estimaría que son necesarias las marcas de atenuación discursiva; en la primera, tal vez por el elevado grado de confianza, se estarían considerando prescindibles-. En concreto, los enunciados escritos se formulan de acuerdo con tres tipos de estructuras:
a. No.
b. No quiero ir al cine.
c. No, no quiero ir al cine contigo. 
En el $65,5 \%$ restante, se localizan marcas de atenuación discursiva. Ahora bien, es preciso distinguir los enunciados en los que se responde explícitamente que no con atenuación de aquellos otros en los que se contesta en el mismo sentido, pero sin usar la partícula discursiva no. Entendemos que, en el segundo caso, el acto verbal es más indirecto que en el primero y, en consecuencia, tanto el grado de atenuación como de cortesía discursivas es mayor.

Cuantitativamente, están prácticamente equiparados; en el 31\% de las ocasiones, se responde mediante la partícula discursiva no, pero atenuando, como ilustran los ejemplos (1), (2) y (3):

(1) No, gracias pero ya tengo planes.

(2) Lo siento, pero no puedo ir contigo.

(3) Tengo una cita, no puedo ir contigo, lo siento.

En el 34,5\% de las respuestas se rechaza la invitación sin usar la partícula discursiva no, como se refleja en (4), (5) y (6):

(4) Lo siento, pero estoy muy preocupada y tengo mucha tarea.

(5) Lo siento mi amiga, pero tengo otros planes, ¿un otro vez?

(6) Yo soy muy ocupada, lo sienta amigo. Nosotros podemos ir otro tiempo.

Como también se observa en los ejemplos (4) a (6), el 41,4\% de los enunciados recurren a la excusa como estrategia atenuadora. En el 20,7\% de los casos, tales excusas incluyen una estructura opositiva introducida por la partícula pero —como en (1), (2), (4) y (5)-. Además, en un $31 \%$, se acompaña una expresión de disculpa (lo siento) —ejemplos (2) a (6) - y, más esporádicamente, en un $17,2 \%$ de los casos, de agradecimiento (gracias) - ejemplo (1)—. En un 20,7\% de los enunciados, encontramos elementos lingüísticos de indeterminación en expresiones del tipo Tengo otro evento / otros planes. Poco habituales, pero también presentes, son la contrapropuesta (6,9\%) — como muestran los ejemplos (5) y (6)-y el uso del apelativo amigo (10,3\%).

\subsubsection{Grupo B1-B2}

Al rechazar una invitación, en un 4,3\% de los casos se responde sin atenuar el enunciado con la partícula discursiva no. En el 56,5\% de las respuestas escritas aparece esta partícula, pero con marcas de atenuación discursiva. Y, en el 39,2\%, se rehúsa la invitación de ir al cine sin recurrir a dicha partícula. 
En las respuestas correspondientes al grupo B1-B2, predomina la estructura formada por los siguientes elementos atenuadores: disculpa + excusa con inicio opositivo, como se observa en (7). En ocasiones, se elide la partícula pero, que encabeza dicho miembro de carácter opositivo - por ejemplo, en (8) - y, en unos pocos casos, se acompaña de una justificación -como en (9) y (10)-:

(7) Lo siento, pero tengo mucha tarea hoy.

(8) Lo siento, no me gusta ir al cine.

(9) Lo siento, pero no puedo ir al cine porque no tengo dinero.

(10) Lo siento, no puedo ir. Ya tengo planes con otra persona.

En términos numéricos, en el $73,9 \%$ de las respuestas, las participantes recurren a la excusa y, en el $52,2 \%$ de los enunciados, se añade a continuación una estructura de tipo opositivo encabezada explícitamente por pero. También un 73,9\% de las respuestas se acompañan de una expresión de disculpa, que, al igual que en el grupo A2-B1, suele ocupar el margen inicial del enunciado (lo siento), y, en muy pocos casos, el final. A diferencia del grupo anterior, en alguna ocasión se sustituye lo siento por perdón, también al comienzo del enunciado (11):

(11) Perdón, pero no puedo ir tengo mucho que hacer.

La expresión de agradecimiento, ahora, solo aparece en el 13\% de los enunciados, y en una ocasión lo hace de forma intensificada (12):

(12) Muchas gracias para invitarme, pero yo no puedo asistir.

En un $21,7 \%$ de las respuestas, aparecen expresiones de indeterminación como recurso de atenuación -en este caso, tener mucho que hacer o mucha tarea son las expresiones preferidas para evadir la invitación; aunque también encontramos no tengo bastante tiempo o tengo *otras planes-. En este grupo, no se proponen alternativas en un $4,3 \%$ de las ocasiones y, con el mismo porcentaje, se emplean apelativos; el enunciado (13) es paradigmático —dado el exceso de atenuadores y marcas de cortesía (disculpa + apelativo + excusa de contenido impreciso introducida por partícula opositiva + segunda excusa + agradecimiento enfático + contrapropuesta en tiempo indeterminado), podría considerarse supercortés-:

(13) Lo siento, mi amigo, pero yo tengo otras planes. También, a mi no me gusta la película que tú verás. jGracias por su invitación! Puedo vamos al cine otra día.

Como novedades igualmente esporádicas, se expresa posibilidad (4,3\%) —*a la mejor - (14) y se usa la interjección ay (4,3\%) (15): 
(14) Lo siento, tengo mucha tarea. A la mejor, la proxima vez.

(15) iAy lo siento, pero necesito hacer tarea!

\subsection{Recursos de atenuación discursiva en el supuesto 2: responder negativa- mente a la petición de un desconocido}

En esta sección, se presta atención al tipo y al número de recursos de atenuación discursiva vinculados a la función comunicativa responder negativamente a una petición, que, ahora, procede de un desconocido: "(2) Un desconocido te pide dinero por la calle. ¿Cómo le dices que no puedes darle nada?”.

\subsubsection{Grupo A2-B1}

El 100\% de las respuestas incluye la partícula discursiva no. Con todo, consideramos que la mayoría de los enunciados niega cortésmente; concretamente, un 62,1\% —así se comprueba en [16] - ; un 31\% no incluye marcas de cortesía ni tampoco de descortesía, conque estimamos que se trata de actos acorteses - por ejemplo, (17)—; finalmente, el 6,9\% restante se considerarían descorteses (18):

(16) No tengo dinero o no puedo dar dollares pero puedo comprar comida para ti.

(17) No puedo dar dinero a ti.

(18) Yo no hablo a la persona.

En este supuesto, las estrategias de atenuación son, sorprendentemente, menos variadas que en el primero. El panorama anterior se simplifica en dos tipos de expresiones atenuadoras que se vinculan a los enunciados base: la excusa y la disculpa. En un 91,3\% de los casos se proporciona una excusa al hecho de no dar dinero a un desconocido, que suele ser no tener dinero. Se diferencian tres tipos de excusas en el corpus analizado; a saber, excusa sin inicio opositivo (60,9\%) (19), excusa con inicio opositivo (17,4\%) (16), y excusa con inicio justificativo (13\%) (20):

(19) No tengo dinero para te doy.

(20) No puedo dar dinero porque no tengo dinero.

En relación con la disculpa, no se distingue ningún subtipo. El 60,9\% de los enunciados incluye esta función comunicativa, que únicamente viene expresada por el exponente funcional lo siento, que, por lo general, ocupa la posición inicial del enunciado, excepto en 
un par de ocasiones, como en (21), ejemplo en el que aparece en posición marginal final y escrito con c:

(21) No tengo dinero, lo siento!

\subsubsection{Grupo B1-B2}

Al contestar negativamente a la petición de una persona desconocida, también en el 100\% de los enunciados se incluye la partícula no. Asimismo, la mayoría de los actos, el 78,3\%, son corteses, frente a un $17,4 \%$ que serían acorteses. Excluimos de este cómputo la respuesta (22) por resultar contradictoria, descortés y cortés al mismo tiempo:

(22) Ignoro él o digo lo siento, no tengo dinero.

Las estrategias de atenuación son exactamente las mismas que identificamos en las producciones escritas del grupo A2-B1. Los porcentajes de las funciones que se realizan en las respuestas del grupo B1-B2 se distribuyen como sigue: en el 82,6\% de las ocasiones se pone una excusa, que vuelve a coincidir con no tener dinero (también, no tener efectivo, no tener cambio o no tener dinero en la bolsa); más específicamente, excusa sin inicio opositivo (47,8\%) (23), excusa con inicio opositivo (30,4\%) (24) y excusa con inicio justificativo $(4,3 \%)(25)$ :

(23) No tengo dinero en mi bolsillo ahora. iLo siento!

(24) Yo quiero, pero no lo tengo.

(25) No puedo donarte dinero porque yo no tengo mucho dinero.

En relación con la disculpa, el 73,9\% de las participantes incorpora esta función en sus respuestas escritas. Como en el primer supuesto (rechazar la invitación de un amigo para ir al cine), en la situación comunicativa que se propone aquí, tanto el grupo A2-B1 como el que nos ocupa muestra predilección por la estructura formada por la suma de las funciones disculpa + excusa con inicio opositivo, que tiende a corresponderse con el enunciado lo siento, pero... Ese exponente, lo siento, no alterna con ningún otro en este caso; en cambio, casi en una cuarta parte de los enunciados que expresan disculpa (23,5\%), dicho exponente aparece en posición marginal final, aunque la tendencia sigue siendo que aparezca en el margen inicial. El ejemplo (26) es paradigmático por cuanto reproduce la construcción prototípica a la que acabamos de hacer referencia y la ubicación del exponente lo siento al principio:

(26) Lo siento, pero yo no tengo nada en este momento. 


\subsection{Comparación de las estrategias de atenuación discursiva entre supues- tos y grupos}

En la tabla 3 se oponen los datos anteriores en términos cuantitativos por porcentajes de aparición.

\section{TABLA 3}

Ítems de atenuación discursiva distribuidos por supuestos y grupos

\begin{tabular}{|c|c|c|c|c|}
\hline \multirow{2}{*}{ ATENUACIÓN DISCURSIVA } & \multicolumn{2}{|c|}{ SUPUESTO 1} & \multicolumn{2}{|c|}{ SUPUESTO 2} \\
\hline & $\mathrm{A} 2-\mathrm{B} 1$ & $\mathrm{B1}-\mathrm{B}_{2}$ & $\mathrm{~A} 2-\mathrm{B}_{1}$ & $\mathrm{~B} 1-\mathrm{B} 2$ \\
\hline$\%$ Actos corteses & 65,5 & 95,7 & 62,1 & 78,3 \\
\hline$\%$ Excusa & 41,4 & 73,9 & 91,3 & 82,6 \\
\hline \% Excusa con inicio opositivo & 20,7 & 52,2 & 17,4 & 30,4 \\
\hline \% Disculpa & 31 & 73,9 & 60,9 & 73,9 \\
\hline \% Agradecimiento & 17,2 & 13 & 0 & 0 \\
\hline \% Indeterminación & 20,7 & 21,7 & 0 & 0 \\
\hline \% Contrapropuestas & 6,9 & 4,3 & 0 & 0 \\
\hline \% Uso de apelativos & 10,3 & 4,3 & 0 & 0 \\
\hline \% Expresión de posibilidad & 0 & 4,3 & 0 & 0 \\
\hline \% Uso de interjección & 0 & 4,3 & 0 & 0 \\
\hline
\end{tabular}

Por un lado, en el gráfico 1 quedan representados los porcentajes relativos a las estrategias de atenuación discursiva empleadas por los grupos A2-B1 y B1-B2 en las respuestas escritas que asocian al supuesto 1.

Por otro lado, el gráfico 2 representa los porcentajes que se refieren a las estrategias de atenuación utilizadas por cada grupo en los enunciados que responden al supuesto 2.

\section{Discusión}

De acuerdo con la primera hipótesis que formulábamos en este trabajo, basándonos en Briz y Albelda (2013), si se tiene en cuenta la mayor proximidad social que se presupone en la situación comunicativa planteada en el supuesto 1 , esperábamos una presencia más escasa de recursos atenuadores en este supuesto que en el segundo, en el que la petición procede de 


\section{GRÁFICO 1}

Uso de estrategias de atenuación discursiva asociadas al supuesto 1 por cada grupo

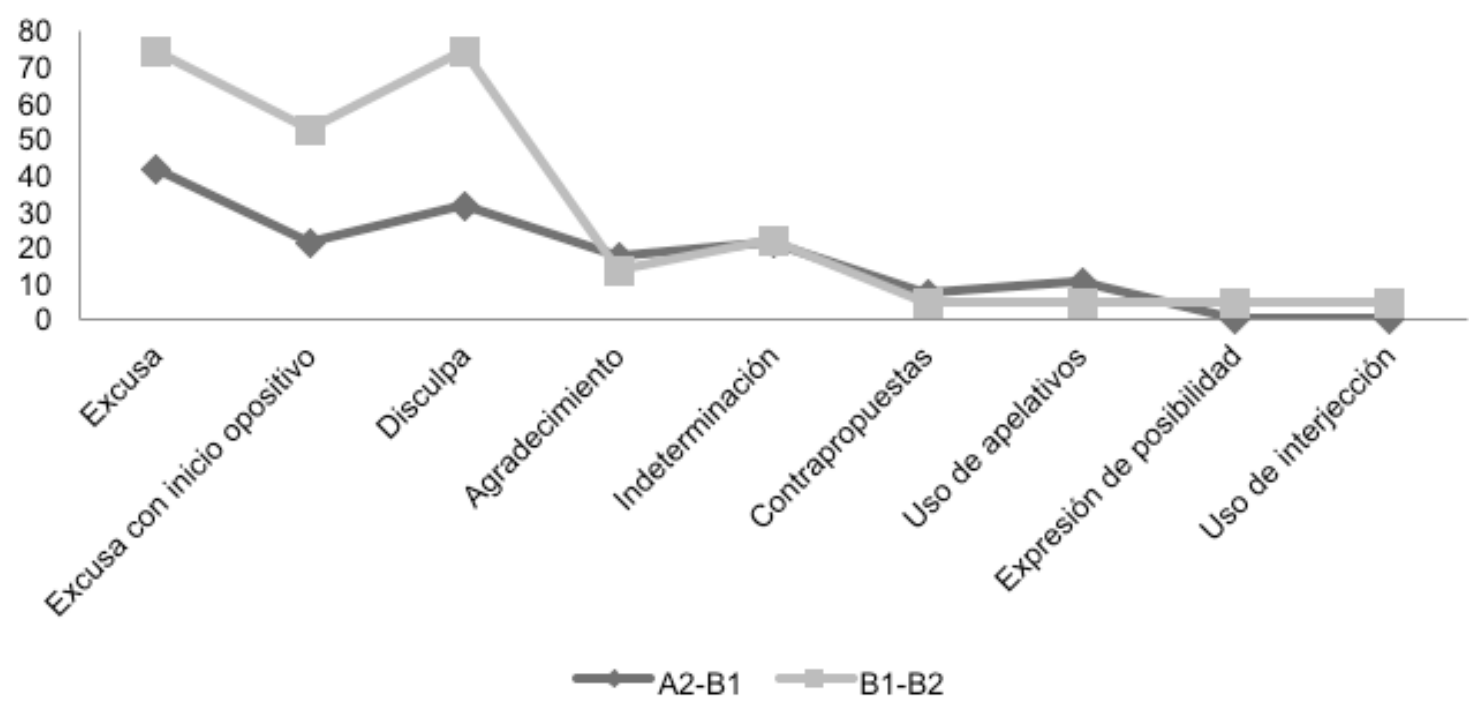

\section{GRÁFICO 2}

Uso de estrategias de atenuación discursiva asociadas al supuesto 2 por cada grupo

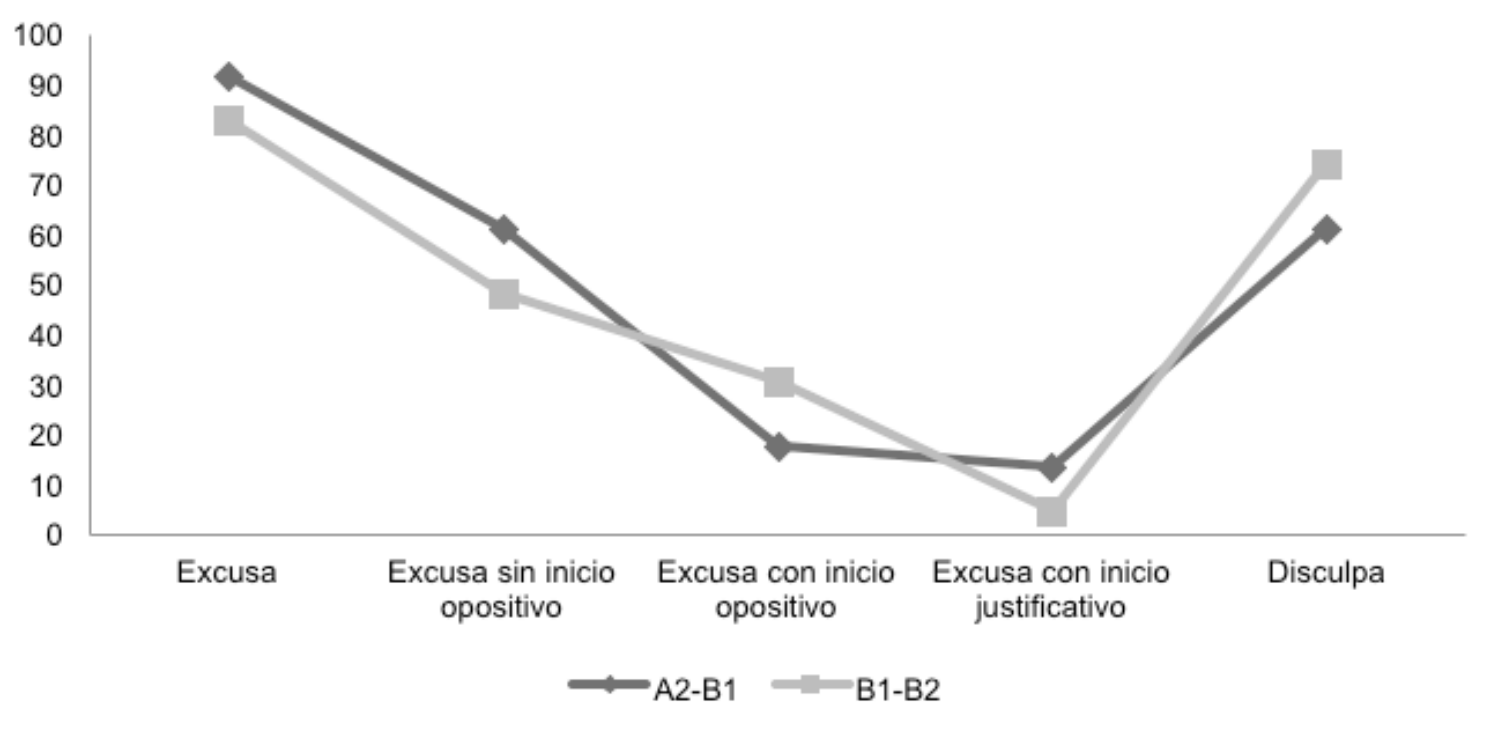

un desconocido. Sin embargo, debemos refutar esta primera hipótesis a la luz de nuestros resultados. Según se ha comprobado aquí, los grupos I (A2-B1) y 2 (B1-B2) coinciden en el hecho de utilizar un mayor número de actos corteses y, en general, de estrategias de cortesía en el primer supuesto que en el segundo, a pesar de que en este último exista una mayor distancia social. Este hecho debe interpretarse en relación con distintos factores básicos: 
- Resulta obvio que no es el mismo acto comunicativo invitar que pedir;

- en tales actos, intervienen factores conceptuales (a qué se invita y qué se pide) y procedimentales (cómo se invita y cómo se pide) que condicionan el modus que adoptan las respuestas de nuestras jóvenes participantes.

Por ende, no solo es crucial la condición de la distancia social entre los interlocutores en los intercambios, sino que intervienen otras de tipo conceptual y procedimental, que están supeditadas al acto comunicativo, que también condiciona per se la mayor o menor presencia de procedimientos de atenuación discursiva en los enunciados, pues ante la petición de dinero por parte de un desconocido, como se planteó en el supuesto 2, las participantes podrían sentirse amenazadas, de modo que podrían no estimar oportuno emplear atenuaciones. Aun así, ambos grupos explotan, tras la negación, un medio pragmático como es la excusa para mitigar las respuestas que proporcionan en sus enunciados escritos, que estructuralmente, en su mayoría, prescinden de inicio opositivo encabezado por pero.

Si atendemos a la segunda hipótesis de nuestra investigación, era previsible que fueran más numerosos y elaborados los recursos modalizadores de atenuación en niveles superiores de dominio lingüístico. Tanto la primera como la segunda consideración, en general, se confirman, aunque han de ser matizadas. Así, los actos corteses que emplea el grupo 2 (B1-B2) son superiores al responder a los supuestos 1 y 2 . Al mismo tiempo, la expresión de la excusa con inicio opositivo y de la disculpa asociadas a ambos supuestos son también superiores en el grupo 2. En cuanto a la mayor elaboración de estos enunciados, el grupo con un mayor nivel de dominio lingüístico (B1-B2), en relación con la disculpa, parece mostrar una mayor conciencia de la movilidad posicional de lo siento, su exponente por excelencia, de sus variantes formales (perdón) y de la estructura y función que, previsiblemente, le suceden: una excusa con inicio opositivo.

En relación con los demás procedimientos de atenuación discursiva, no podemos generalizar una pauta única. No obstante, en el supuesto 1 , la expresión del agradecimiento, la propuesta de alternativas o el uso de apelativos son cuantitativamente superiores en las respuestas del grupo 1. La indeterminación en la información proporcionada es superior, sin embargo, en el grupo 2, que, además, utiliza dos estrategias atenuadoras nuevas: Ia expresión de la posibilidad y la interjección.

A pesar de que trascienda los objetivos de este trabajo, conviene poner siquiera muy brevemente estos resultados en relación con el contenido del PCIC en torno a las funciones (1) rechazar una invitación y (2) responder a una petición negándose a su cumplimiento — que, en este último caso, distingue de forma cortés y de forma tajante-. En el nivel A2, para rechazar invitaciones, se consignan dos opciones: el agradecimiento y la disculpa, que son, en efecto, los procedimientos pragmáticos que con mayor frecuencia se aplican, pues es habitual que 
la excusa vaya precedida, precisamente, de una disculpa. Los exponentes funcionales gracias - sus variantes intensificadas muchas/muchísimas gracias son poco habituales-y lo siento son los más frecuentes en nuestra muestra y coinciden con los que incluye el PCIC en el nivel A2. Las excusas, presentes en los niveles A2, B1 y B2 del PCIC, concuerdan igualmente con nuestros datos. La contrapropuesta, que aparece mencionada solo en el nivel B2 del PCIC, es utilizada tanto por el grupo 1 como por el 2, pero no es una estrategia productiva.

La segunda función, negarse al cumplimiento de una petición, es respondida, por lo común, de forma cortés por los dos grupos de participantes, a pesar de que existen ejemplos en los que se contesta de forma tajante. Nuevamente, los enunciados que analizamos coinciden con los exponentes que incorpora el PCIC en los niveles A2 a B2: Lo siento..., para la disculpa, y pero..., para introducir una excusa — la partícula es que está ausente en nuestras muestras-.

Por último, resulta conveniente referirnos a las particularidades que supone la realización de un estudio sobre estrategias de atenuación discursiva a partir de un instrumento de recogida de datos basado en una tarea de expresión escrita. Una de las principales limitaciones viene impuesta por la falta de contextualización que presentan las preguntas que fueron planteadas por escrito a las alumnas de ELE. Más allá de la formalidad/informalidad de la situación o del conocimiento/desconocimiento del emisor, por un lado, se pierde información procedimental asociada a las cuestiones (cómo se invita y cómo se pide), ya que no se provee en el cuestionario una formulación concreta de la invitación (supuesto I) ni de la petición (supuesto 2) (cortesía codificada), y, por otro lado, la modalidad escrita es incapaz de reflejar los valores contextuales corteses o descorteses que podría transmitir, por ejemplo, la entonación de los enunciados (cortesía interpretada). Con todo, aun habiendo proporcionado un enunciado codificado como cortés o descortés, fuera de su contexto discursivo, la cortesía codificada no siempre coincide con la cortesía interpretada (Briz, 2004). En suma, en el presente estudio no hemos podido exceder los límites del enunciado como nivel de análisis y esto ha impedido que hayan podido incorporarse de una manera más amplia y efectiva los elementos que intervienen en el contexto, lo que, sin más remedio, restringe las posibilidades interpretativas y de respuesta de las participantes a las situaciones que se exponen en el instrumento escrito.

\section{Conclusiones}

El estudio ha confirmado que los procedimientos de atenuación discursiva empleados por alumnas norteamericanas para rechazar una invitación y negarse a una petición cumplen, en general, con las directrices del PCIC para los niveles de dominio indicados.

El hecho de que los exponentes funcionales gracias y sus variantes intensificadas muchas/muchísimas gracias sean poco frecuentes en las respuestas obtenidas de negación de 
una invitación, frente a lo siento, que predomina entre todas las variantes, es solo uno de los ejemplos que nos lleva a considerar, por una parte, las interferencias de la lengua materna (en este caso, la estructura I'm sorry para declinar una invitación) y, por otra, las bases de la pragmática intercultural. La presencia o ausencia de agradecimiento corresponden a patrones culturales en la lengua y cultura de origen. Hay que considerar también las diferencias entre el espacio interpersonal en las culturas de contacto (mediterráneos, árabes y africanos) y las de no contacto (norteamericanos, anglosajones, escandinavos y asiáticos) respecto a lo que se considera "normal" en cada cultura. Si, en el supuesto 2 de la investigación, el desconocido hubiera pedido información, y no dinero, las participantes norteamericanas en el estudio no se habrían sentido amenazadas y quizás hubieran usado más mecanismos de atenuación en el caso de no disponer de la información. En este sentido, los resultados de la investigación se deberían interpretar considerando también las aportaciones de la pragmática contrastiva y la intercultural, a pesar de que los dos supuestos planteados en el estudio no presentan notables diferencias culturales.

La opción de ser estratégicamente cortés en el primer supuesto y no sentir esta necesidad en el segundo marca las diferencias en los mecanismos y en el grado de atenuación.

Los dos grupos en el estudio utilizan un mayor número de actos corteses en el primer supuesto que en el segundo, a pesar de que en este último exista una mayor distancia social. Tal como hemos señalado anteriormente, influye la diferencia entre los actos comunicativos en sí -invitar y pedir - y, además, intervienen factores conceptuales (a qué se invita y qué se pide) y procedimentales (cómo se invita y cómo se pide). En consecuencia, creemos que el estudio aporta resultados sorprendentes y relevantes a la vez al quedar refutada la primera hipótesis, según la cual a una mayor distancia social le correspondería un mayor uso de atenuantes. Sí queda validada la segunda hipótesis sobre un mayor uso de la atenuación y una mejor expresión en niveles superiores de dominio lingüístico. Sin embargo, resulta llamativo el dato de que en el grupo de nivel inferior se observa mayor uso del agradecimiento y de propuestas de alternativas.

En futuras investigaciones, nos planteamos comparar los resultados obtenidos con los de sujetos masculinos para contrastarlos por sexo, así como aumentar el rango de las variables edad y nacionalidad con el fin de examinar los procedimientos de atenuación discursiva en el marco de la pragmática intercultural. Además, ampliaremos el estudio sobre los procedimientos atenuadores a cuatro funciones más que fueron planteadas, a partir de distintos supuestos, en el cuestionario que diseñamos para esta investigación: dar una opinión, valorar, pedir un objeto y pedir un favor. Por último, sería muy oportuno completar los datos sobre los mecanismos de atenuación que emplean los estudiantes de ELE con los relativos a las estrategias de intensificación que asocian a las mismas funciones mediante el uso de diversas marcas modales como las de evidencia o las de sinceridad (González Ruiz, 2005), entre otras. 


\section{Bibliografía citada}

Albelda, Marta, 2013: “La atenuación: tipos y estrategias” en José Ramóm Gómez Molina (coord.): El español de Valencia. Estudio sociolingüístico, Frankfurt: Peter Lang, 315-343.

Albelda, Marta, y Ana M. Cestero, 2011: "De nuevo, sobre los procedimientos de atenuación lingüística”, Español Actual. Revista de Español Vivo 96, 9-40.

Albelda, Marta, Antonio Briz, Ana M. Cestero, Dorota Kotwica y Cristina Villalba, 2014: "Ficha metodológica para el análisis pragmático de la atenuación en corpus discursivos del español. (ES. POR.ATENUACIÓN)", Oralia 17, 7-62.

BRIz, Antonio, 2004: "Cortesía verbal codificada y cortesía verbal interpretada en la conversación” en Diana Bravo y Antonio Briz (eds.): Pragmática sociocultural: estudios sobre el discurso de cortesía en español, Barcelona: Ariel, 67-93.

BRIz, Antonio, 2012: "La atenuación lingüística: propuesta metodológica para su análisis”, conferencia presentada en el Congresso Internacional Interdisciplinar Cortesia: Olhares e (Re) invenções, Lisboa: Universidade Aberta.

Briz, Antonio, y Marta AlBelda, 2013: “Una propuesta teórica y metodológica para el análisis de la atenuación lingüística en español y portugués. La base de un proyecto en común (ES.POR. ATENUACIÓN)", Onomázein 28, 288-319.

Cestero, Ana M., 2012: "Recursos lingüísticos de atenuación en el habla de Madrid: estudio sociopragmático” en Tomás Jiménez JuliÁ, Belén López Meirama, Victoria Vázquez Rozas y Alexandre Veiga Rodriguez (eds.): Cum corde et in nova grammatica. Estudios ofrecidos a Guillermo Rojo, Santiago de Compostela: Universidad de Santiago de Compostela, 233-246.

Consejo de Europa, 2002 [2001]: Marco común europeo de referencia para las lenguas: aprendizaje, enseñanza, evaluación, Madrid: Instituto Cervantes, Ministerio de Educación, Cultura y Deporte, y Anaya.

Félix-Brasdefer, J. César, 2004: "La mitigación en el discurso oral de mexicanos y aprendices de español como lengua extranjera” en Diana Bravo y Antonio Briz (eds.): Pragmática sociocultural: estudios sobre el discurso de cortesía en español, Barcelona: Ariel, 285-299.

GonzÁlez Ruiz, Ramón, 2005: "Modalizadores de sinceridad, cooperación y estrategias comunicativas”, Anuario de Lingüística Hispánica 21-22, 199-228.

Gómez Mesas, María, y Francisco Rodriguez Muñoz, 2014: "La presencia del adverbio en el sujeto: sustantivación y modalidad”, Signos Lingüísticos 10 (19), 132-143.

Hidalgo Navarro, Antonio, 2006: "La expresión de cortesía en español hablado. Marcas y recursos prosódicos para su reconocimiento en la conversación coloquial” en Milka Villayandre (ed.): 
Actas del XXXV Simposio Internacional de la Sociedad Española de Lingüística, León: Universidad de León, 957-979.

Instituto Cervantes, 2006: Plan curricular del Instituto Cervantes. Niveles de referencia para el español, Madrid: Instituto Cervantes.

Kebrat-Orecchioni, Catherine, 2004: “¿Es universal la cortesía?” en Antonio Briz y Diana Bravo (eds.): Pragmática sociocultural: estudios sobre el discurso de cortesía en español, Barcelona: Ariel, 39-54.

Llamas Saiz, Carmen, 2010: "Competencia discursiva escrita en los niveles Avanzado (B2) y Dominio (C1): Ia unidad textual en los manuales de ELE", MarcoELE: Revista de Didáctica Español Lengua Extranjera 11, 1-25.

Lyons, John, 1977: Semantics, vol. 2, Londres: Cambridge University Press.

Martin Peris, Ernesto, Encarnación Atienza Cerezo, Maximiano Cortés Moreno, María Vicente González Argüello, Carmen lópez Ferrero y Sergi Torner Castells, 2008: Diccionario de términos clave de ELE, Madrid: SGEL.

Mendiluce, Gustavo, 2005: Estudio comparado inglés/español del discurso biomédico escrito: la secuenciación informativa, la matización asertiva y la conexión argumentativa en la introducción y la discusión de artículos biomédicos escritos por autores nativos y no-nativos. Tesis doctoral, Valladolid: Universidad de Valladolid.

NejSTRÖm, Emelie, 2012: El uso de atenuaciones en español entre alumnos suecos del bachillerato. Rechazos, consejos y peticiones, Universidad de Estocolmo: Digitala Vetenskapliga Arkivet.

NíkleVa, Dimitrinka, 2015: "La cortesía en los correos electrónicos de estudiantes universitarios como parte de la competencia pragmático-discursiva”, Spanish in Context 12 (2), 280-303.

Oesterreicher, Wulf, 1996: "Lo hablado en lo escrito. Reflexiones metodológicas y aproximación a una tipología” en Thomas Kostchi, Wulf Oesterreicher y Klaus Zimmermann (eds.): El español hablado y la cultura oral en España e Hispanoamérica, Frankfurt/Madrid: Vervuert/Iberoamericana, 317-340.

Ramos GonzÁlez, Noelia, 2015: La expresión de la cortesía en el aula de ELE. Una propuesta didáctica para evitar el fallo pragmático a partir del análisis de las series de Televisión Española. Tesis doctoral, Granada: Universidad de Granada.

Reguelro, María Luisa, y Daniel Sáez, 2015: El español académico. Guía práctica para la elaboración de textos académicos, Madrid: Arco/Libros.

Rodriguez Muñoz, Francisco, 2009: "Estudio sobre las funciones pragmadiscursivas de ¿no? y ¿eh? en el español hablado”, Revista de Lingüística Teórica y Aplicada 47 (1), 83-101. 
Rodriguez Muñoz, Francisco, 2012: "Neutralización, remodalización o subjetivización? A propósito de la combinación ‘muy + adjetivo diminutivo’ en español”, Revista Española de Lingüística Aplicada 25, 211-224.

Traugott, Elizabeth, 1989: "On the rise of epistemic meanings in English: an example of subjectification in semantic change", Language 65 (1), 31-55.

\section{Agradecimientos}

Los autores de este artículo desean agradecer la colaboración del Prof. D. Francisco Secci, que desarrolla su actividad docente en The Spence School (Nueva York, Estados Unidos). Sin su ayuda, habría sido imposible recoger los datos que han servido de base para realizar este estudio. 